\title{
EVALUATION OF BONDING ORBITALS IN AMORPHOUS SILICON BY MEANS OF THE CHEMICAL PSEUDOPOTENTIAL METHOD
}

\author{
M.A. GRADO CAFFARO, consultant (Madrid, Spain) and \\ M. GRADO CAFFARO, consultant (Madrid, Spain) \\ C./ Julio Palacios, 11, 9-B, 28029-Madrid (Spain)
}

(Received August 12, 1993; in final form September 10, 1993)

The chemical pseudopotential method has been used by a number of workers in order to study the valence bands of amorphous tetrahedrally bonded semiconductors. However, various problems related to this method are unsolved. In this paper, a theoretical formulation tending to clarify some of these problems is presented.

This formulation concerns bonding orbitals and is valid, in principle, for amorphous silicon.

\section{INTRODUCTION}

Localised pseudopotential theory is applicable to evaluate localised orbitals in amorphous semiconductors. This theory was applied by P.W. Anderson ${ }^{1,2}$ in order to calculate localised orbitals for use in solid-state chemical bonding problems. In addition, other workers have studied bonding problems in the context of amorphous semiconductors. ${ }^{3}$

The chemical pseudopotential method is related to certain empirical and semiempirical techniques as, for example, the empirical pseudopotential method (EPM). In certain cases, EPM has provided results in good agreement with experimental results. This method, as well as other similar methods, have been used to analyze complex systems. ${ }^{4-7}$

In the following, we shall use a method that offers a high level of reliability. By considering bonding orbitals, we shall establish an interesting equation for the electronic density of states corresponding to amorphous silicon. In fact, our theoretical formulation involves the determination of the electronic density of states in a unique way.

\section{THEORETICAL FORMULATION}

First, let us consider two isolated atoms 1 and 2. We can write:

$\mathrm{H}_{12}\left|\phi_{12}\right\rangle=\mathrm{E}\left|\phi_{12}\right\rangle$ 
Where $\mathrm{H}_{12}$ denotes hamiltonian, $\mathrm{E}$ means energy, and $\left|\phi_{12}\right\rangle$ is local bonding orbital. A given electron in the bonding orbital is influenced by the potential of perturbation of the above atoms. This potential satisfies the following relation:

$\mathrm{V}_{12}=\mathrm{H}-\mathrm{H}_{12}$

where $V_{12}$ is the potential and $H$ is the total single-electron hamiltonian.

We shall formulate the potential by using the Born model. Then, we have: ${ }^{8}$

$\mathrm{V}_{12}=\frac{3}{2} \beta\left[\left(\overrightarrow{\mathrm{u}}_{1}-\overrightarrow{\mathrm{u}}_{2}\right) \cdot \hat{\mathrm{r}}_{12}\right]^{2}+\frac{1}{2}(\alpha-\beta)\left|\overrightarrow{\mathrm{u}}_{1}-\overrightarrow{\mathrm{u}}_{2}\right|^{2}$

where $\overrightarrow{\mathrm{u}}_{1}$ and $\overrightarrow{\mathrm{u}}_{2}$ are the displacement vectors of the atoms 1 and 2 respectively, and $\hat{r}_{12}$ stands for a unit vector that connects the atoms. The parameters $\alpha$ and $\beta$ are force constants.

On the other hand, we choose $\alpha=2 \beta$ and we consider $\overrightarrow{\mathrm{u}}_{1} \cdot \hat{\mathrm{r}}_{12}=\overrightarrow{\mathrm{u}}_{2} \cdot \hat{\mathrm{r}}_{12}$ (see ref. [9]). Then, from eq. (3) it is obtained:

$\mathrm{V}_{12}=\frac{1}{2} \beta\left|\overrightarrow{\mathrm{u}}_{1}-\overrightarrow{\mathrm{u}}_{2}\right|^{2}$

By combining eqs. (1), (2) and (4), we have:

$2 \mathrm{H}\left|\phi_{12}\right\rangle-\beta\left|\overrightarrow{\mathrm{u}}_{1}-\overrightarrow{\mathrm{u}}_{2}\right|^{2}\left|\phi_{12}\right\rangle=2 \mathrm{E}\left|\phi_{12}\right\rangle$

Moreover, the molecular orbital in eq. (5) can be expressed as follows: ${ }^{3}$

$\left|\phi_{12}\right\rangle=\mathrm{c}\left(\left|\phi_{1 \rightarrow 2}\right\rangle+\left|\phi_{2 \rightarrow 1}\right\rangle\right)$

where $\mathrm{c}$ is a constant, $\left|\phi_{1 \rightarrow 2}\right\rangle$ is the orbital on atom 1 directed towards atom 2 , and $\left|\phi_{2 \rightarrow 1}\right\rangle$ is the orbital on atom 2 directed towards atom 1. Eq. (6) represents the normalized sum of a Pauling-type hybridised $\mathrm{sp}^{3}$ orbital on each atom.

Finally, we will consider the local electronic density of states namely: ${ }^{10}$

$g(\overrightarrow{\mathrm{r}}, \mathrm{E})=\sum_{\mathrm{n}}\left[\left|\int_{R^{\prime}} \psi_{\mathrm{n}}\left(\overrightarrow{\mathrm{r}}^{\prime}\right) \phi_{12}\left(\overrightarrow{\mathrm{r}}^{\prime}-\overrightarrow{\mathrm{r}}\right) \mathrm{d} \overrightarrow{\mathrm{r}}^{\prime}\right|^{2} \delta\left(E-\mathrm{E}_{\mathrm{n}}\right)\right]$

where $\psi_{\mathrm{n}}$ denotes eigenfunction that satisfies: ${ }^{3}$

$\mathrm{H}\left|\psi_{\mathrm{n}}\right\rangle=\mathrm{E}_{\mathrm{n}}\left|\psi_{\mathrm{n}}\right\rangle$

In eq. (8) $E_{n}$ is the energy eigenvalue corresponding to $\psi_{n}$. By determining $\psi_{n}$ from eq. (8), it is feasible to use eq. (7) by also taking into consideration eqs. (5) and (6). Furthermore, for very small clusters of atoms, we can use the following approximation to obtain the total density of states, namely: ${ }^{11}$

$\mathrm{g}(\mathrm{E}) \approx \Omega \mathrm{g}(\overrightarrow{\mathrm{o}}, \mathrm{E})$ 
where $\Omega$ is the volume of the cluster and $g(\vec{o}, E)$ arises from eq. (7) so that:

$\mathrm{g}(\mathrm{E}) \approx \Omega \sum_{\mathrm{n}}\left[\left|\int_{\mathrm{R}^{\prime}} \psi_{\mathrm{n}}\left(\overrightarrow{\mathrm{r}}^{\prime}\right) \phi_{12}\left(\overrightarrow{\mathrm{r}}^{\prime}\right) \mathrm{d} \overrightarrow{\mathrm{r}}^{\prime}\right|^{2} \delta\left(\mathrm{E}-\mathrm{E}_{\mathrm{n}}\right)\right]$

\section{DISCUSSION}

We have established a chain of equations whose end is the total electronic density of states for amorphous silicon. This total density arises from the local density of states. All equations exposed here are important; in particular, eq. (8) is the Schrödinger equation corresponding to electronic wave functions.

In addition, the consideration of the Born model in order to describe the interaction of two isolated atoms constitutes an important fact. This model has been applied the last twenty years with limited success.

\section{REFERENCES}

1. P.W. Anderson. Phys. Rev. Lert. 21 (1968), 13.

2. P.W. Anderson. Phys. Rev. 181 (1969), 25.

3. D.W. Bullett. AIP Conf. Proc. n 20 (1974), 139-144.

4. J.D. Joannopoulos, M.L. Cohen. AIP Conf. Proc. n- 20 (1974), 85-89.

5. J.D. Joannopoulos, F. Ynduráin. Phys. Rev. B 10 (1974), 5164.

6. J. Avendaño, F.L. Castillo-Alvarado, R.A. Barrio. J. Non-Cryst. Solids 137-138 (1991), 299302.

7. K. Pierz, W. Fuhs, H. Mell. Phil. Mag. B 63 (1991), 123.

8. M.F. Thorpe. AIP Conf. Proc. n 20 (1974), 267-273.

9. R. Alben. AIP Conf. Proc. no 20 (1974), 255.

10. R. Haydock, V. Heine, M.J. Kelly. J. Phys. C 5 (1972), 2845.

11. M.A. Grado, M. Grado: "A new mathematical model for cluster calculation in tetrahedrally bonded amorphous semiconductors." Act. Pass. Electronic Comp., in press, (1993). 

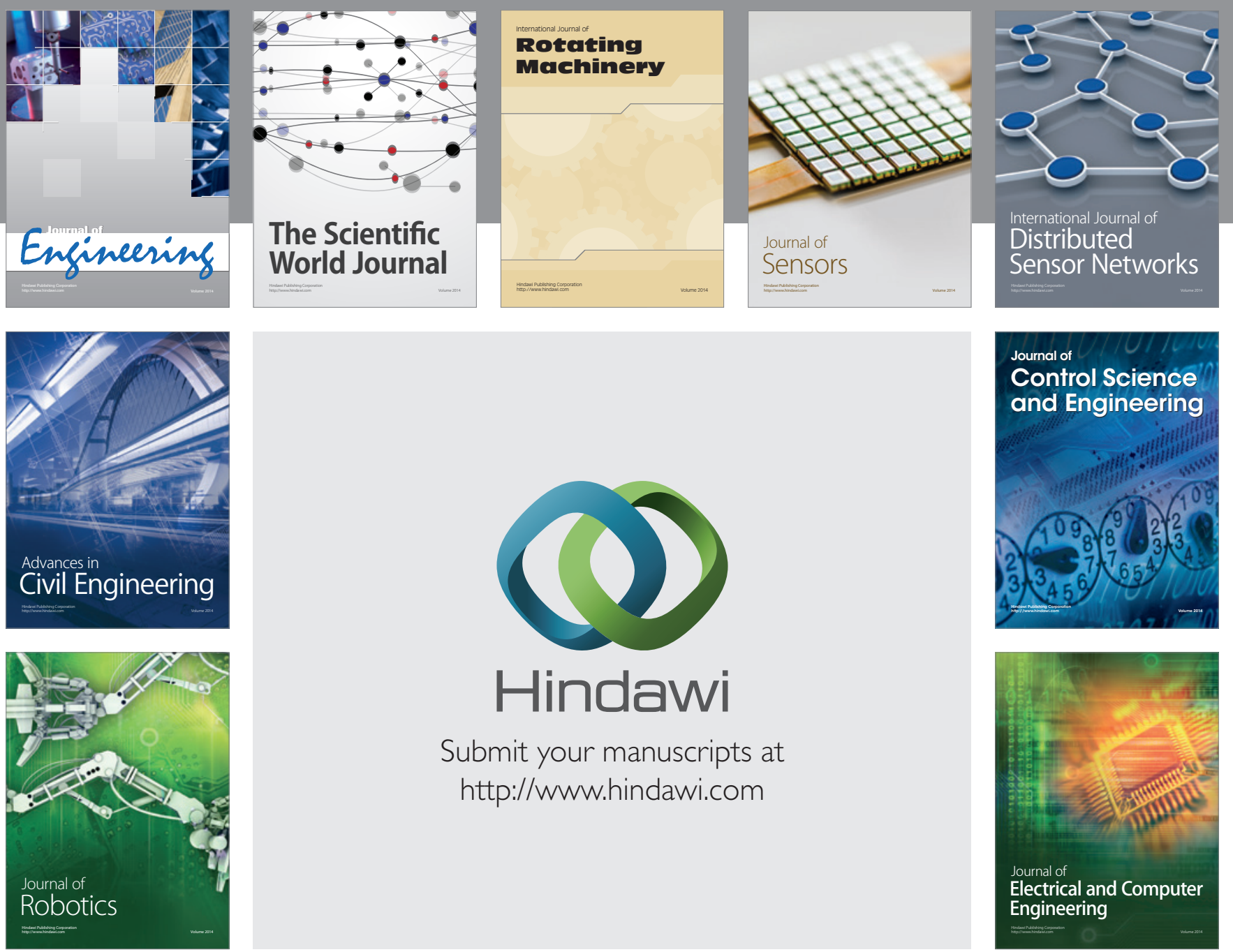

Submit your manuscripts at

http://www.hindawi.com
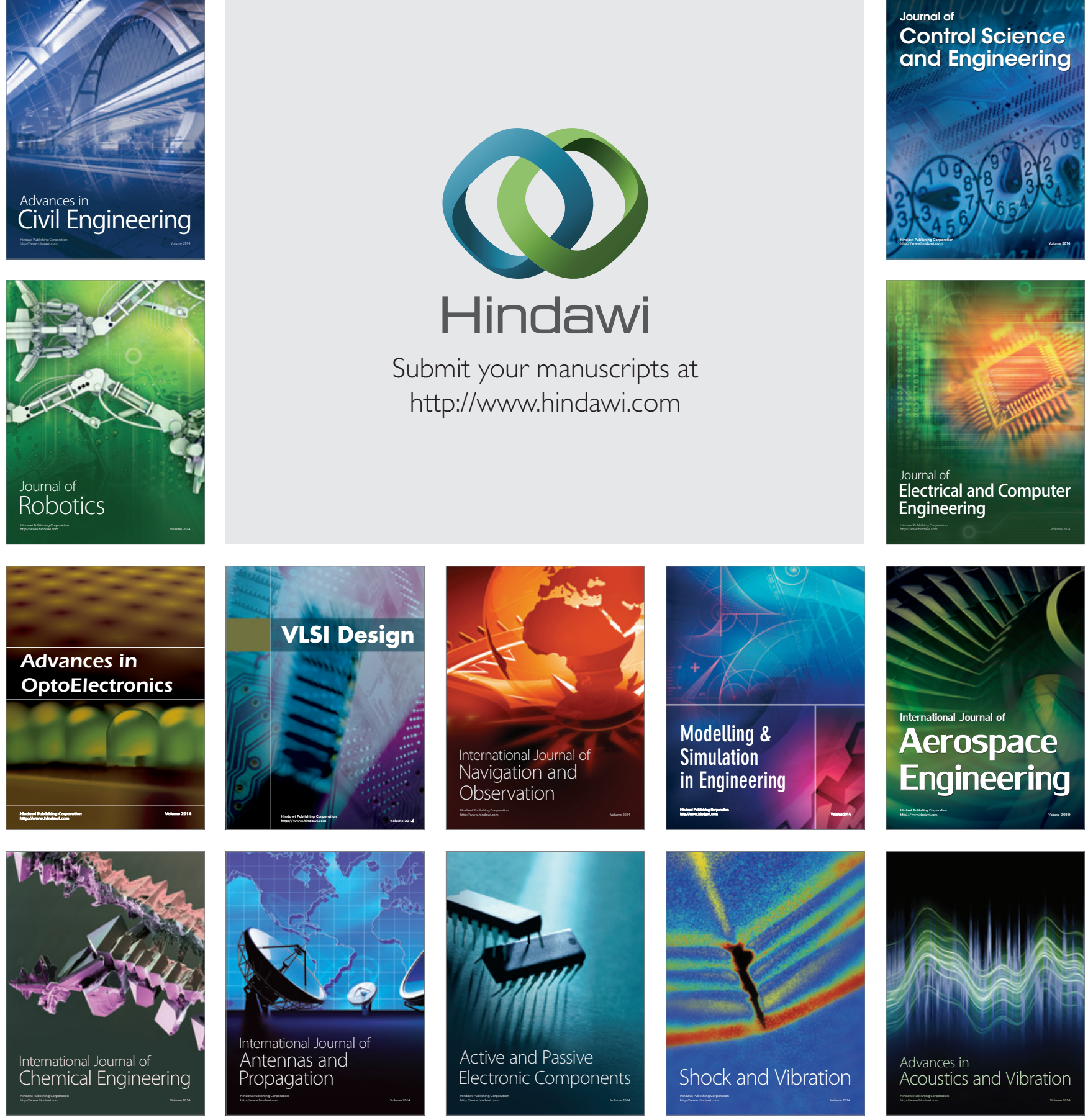